\title{
EL POTENCIAL DE LA CORRESPONSABILIDAD EN EL ÁMBITO UNIVERSITARIO: UNA EXPERIENCIA DE TRABAJO COLABORATIVO DESDE LA BIBLIOTECA PARA LA FORMACIÓN EN LA PREVENCIÓN DE LA VIOLENCIA DE GÉNERO
}

\author{
Clara Isabel Rincón Muñoz* \\ Biblioteca de la Facultad de Ciencias Económicas y Empresariales. Universidad de Valladolid. \\ Azucena Stolle Arranz ${ }^{* *}$ \\ Biblioteca de la Facultad de Ciencias Económicas y Empresariales. Universidad de Valladolid. \\ Carmen Rodríguez Sumaza*** \\ Departamento de Sociología y Trabajo Social. Universidad de Valladolid.
}

\begin{abstract}
Resumen: El presente trabajo aborda el tema de la corresponsabilidad desde una doble perspectiva. En primer lugar, se reflexiona sobre la importancia de la corresponsabilidad en el ámbito de las organizaciones de enseñanza superior enmarcadas en el modelo educativo impulsado por el Proceso de Bolonia. En este sentido, se entiende que una universidad de calidad es, entre otras cuestiones, una organización donde todos sus miembros (personal docente e investigador, personal de administración y servicios y alumnado) y todos los recursos disponibles se implican de manera más activa, coordinada y comprometida en los procesos de aprendizaje del alumnado.

En segundo lugar se presenta una propuesta concreta de trabajo colaborativo para promover una formación de carácter transversal en materia de corresponsabilidad como estrategia para la prevención de la violencia de género impulsada desde el servicio de biblioteca en estrecha colaboración con el personal docente e investigador y dirigida a estudiantes de grado y a toda la comunidad universitaria en general.

Palabras clave: Corresponsabilidad; prevención de la violencia de género; bibliotecas universitarias; formación.
\end{abstract}

Title: THE POTENTIAL OF CO-RESPONSIBILITY IN THE ACADEMIC SPHERE: A COLLABORATIVE WORKING EXPERIENCE FROM LIBRARY SERVICES TO PREVENT GENDER-BASED VIOLENCE.

Abstract: This paper addresses the subject of co-responsibility from a dual perspective. Firstly, we reflect on the importance of co-responsibility in the field of higher education organizations framed in the educational model promoted by the Bologna process and argue that University's quality requires an organization where all members (teachers, researchers, staff and students) and all available resources are more actively, coordinately and committedly involved in the students learning processes.

Secondly, we submit a specific proposal for undergraduate students based on collaborative work between library staff, teachers and researchers to promote cross-curricular training on co-responsibility as a way to prevent genderbased violence.

Keywords: Co-responsibility; gender-based violence prevention; university libraries; education.

\section{INTRODUCCIÓN}

El término corresponsabilidad es extraordinariamente complejo y versátil. Susceptible de ser aplicado a muy distintas cuestiones o en muy diferentes ámbitos de la vida, en términos generales viene a hacer referencia a la existencia de niveles compartidos de responsabilidad en la ocurrencia de un hecho por parte de diferentes personas, agentes, instituciones, etc. En materia de igualdad entre mujeres y hombres, y en el contexto de los modelos de estado del bienestar propios de nuestra sociedad europea actual, se entiende que la igualdad es un bien social al que pueden y deben contribuir todos los agentes (Estado, mercado, familias, tercer sector) con el fin de erradicar las conductas discriminatorias y promover las condiciones para que unos y otras puedan desarrollarse plenamente como personas y ejercer adecuadamente sus derechos sociales.

En el largo camino que aún separa la igualdad teórica de la igualdad real de oportunidades entre hombres y mujeres persisten aún muy diversos ámbitos en los que resulta necesario reforzar los compromisos para conseguir una distribución más equilibrada entre los recursos y en las relaciones. Así, la corresponsabilidad en el ámbito familiar y en el ámbito de las organizaciones del trabajo se constituyen -entre otros- en ejes estratégicos de incidencia,

\footnotetext{
* claraisabel.rincon@uva.es

** azucenamaria.stolle@uva.es

***sumaza@eco.uva.es
}

Recibido: 09-06-2014; aceptado: 08-10-2014.

RINCÓN MUÑOZ, C.I.; STOLLE ARRANZ, A. y RODRÍGUEZ SUMAZA, C. El potencial de la corresponsabilidad en el ámbito universitario: una experiencia de trabajo colaborativo desde la biblioteca para la formación en la prevención de la violencia de género. Anales de Documentación, 2014, vol. 17, n 2. Disponible en: http://dx.doi.org/10.6018/analesdoc.17.2.200211 
entendiendo que siguen siendo precisos todo tipo de esfuerzos encaminados a conseguir, por un lado, una distribución más equitativa de las responsabilidades y las tareas que se realizan dentro y fuera del hogar y, por otro, a lograr unas relaciones laborales más saludables basadas verdaderamente en el respeto, la autonomía y el mutuo reconocimiento entre mujeres y hombres. Otro ámbito de gran relevancia social es el de la violencia de género, una lacra que para su definitiva erradicación requiere de la colaboración de muy diversas instancias, entre las que las instituciones educativas en todos sus niveles están llamadas a jugar un papel clave en su prevención dado su relevante papel como agentes de socialización.

Siendo como es todavía la igualdad real una meta a conseguir y perfilándose la corresponsabilidad como una estrategia idónea para avanzar en esa dirección, el corolario consecuente es la necesidad de generar una cultura de corresponsabilidad que permita visibilizar y denunciar las desigualdades existentes y avanzar desde y con la diferencia hacia un nuevo modelo de ciudadanía y de sociedad. Para la promoción de este cambio cultural es necesario educar en valores igualitarios y solidarios y uno de los contextos idóneos para ello son los espacios universitarios ${ }^{1}$, entendiendo por ellos contextos que trascienden el aula, lugar de enseñanza por excelencia, y que pueden abarcar diversos servicios y actividades que se prestan habitualmente a la comunidad universitaria.

Partiendo de las anteriores premisas, el tema de la corresponsabilidad se aborda en este trabajo desde una doble perspectiva. En primer lugar, se reflexiona sobre la importancia de la corresponsabilidad en el ámbito de las organizaciones de enseñanza superior, en el marco del modelo educativo impulsado por el Proceso de Bolonia. En este sentido, se entiende que una universidad de calidad es, entre otras muchas cuestiones, una organización donde todos sus miembros y todos los recursos disponibles se implican de manera más activa, coordinada y comprometida en los procesos de aprendizaje del alumnado, que pasan a ser el verdadero centro de atención. Este cambio, que está obligando a redefinir los papeles a desarrollar por los miembros de la institución universitaria (personal docente e investigador -PDI-, personal de administración y servicios -PAS- y estudiantes), también afecta a sus estructuras y servicios. En este contexto, los servicios de biblioteca vienen a ocupar una nueva posición estratégica en continua evolución, desde el mero "almacén de libros” a verdaderos "centros de recursos para el aprendizaje y la investigación”, lo que supone una creciente necesidad de comunicación e interacción entre el PAS, el PDI y el alumnado. En segundo lugar, se desarrolla la idea de que el ejercicio de la responsabilidad social universitaria pasa necesariamente, y entre otras cuestiones, por el desarrollo de estrategias que permitan contribuir de todas las maneras posibles a la promoción de valores que, como la corresponsabilidad, promuevan modelos de ciudadanía y sociedad democráticos, respetuosos e igualitarios.

A partir de estos presupuestos se presenta una propuesta concreta de acción formativa elaborada a partir de una experiencia de trabajo colaborativo entre personal del servicio de biblioteca y personal docente e investigador para promover la sensibilización y la formación en materia de prevención de la violencia de género de estudiantes universitarios de los grados de Marketing e Investigación de Mercados y de Periodismo de la Universidad de Valladolid (UVa), así como un taller formativo para el manejo de la herramienta Dialnet que busca, a la vez que el desarrollo de competencias informacionales, acercar a la comunidad universitaria a la citada temática. Estas propuestas son fruto de la participación a lo largo del año 2013 del personal de biblioteca en el proyecto de investigación "Seminario iberoamericano de formación en corresponsabilidad” (SIFOC)², desde el que se abordó la formación en valores en el ámbito universitario para la prevención de la violencia de género.

\section{LA CORRESPONSABILIDAD COMO VALOR EDUCATIVO Y COMO ESTRATEGIA DE TRABAJO EN EL ÁMBITO DE LAS ENSEÑANZAS UNIVERSITARIAS}

Las universidades europeas y españolas tienen ante sí un importante desafío en el contexto de la actual sociedad del conocimiento. Por un lado, y así nos lo han transmitido los responsables de la política educativa que se desencadena con el Proceso de Bolonia, está el reto de contribuir a formar personas competentes capaces de dar respuesta a los requerimientos técnicos y científicos cada vez más complejos y diversos que el momento actual exige. Por otro lado, se espera que sirvan de instrumentos para el desarrollo local y regional y para la consolidación de la cohesión social; es decir, que actúen como catalizadores del cambio hacia un modelo de sociedad sostenible, responsable y comprometida con el progreso social. ${ }^{3}$

Las reformas de los planes de estudio a las que hemos asistido en los últimos años han pretendido, entre otras cuestiones, reorganizar los conocimientos y adecuar los contenidos disciplinares a los nuevos perfiles profesionales. En paralelo, la vigente ley de universidades ${ }^{4}$ nos recuerda en su propio preámbulo "la importancia de la universidad como transmisor esencial de valores”, destacando la necesidad de contribuir de esta manera a "alcanzar una sociedad tolerante e igualitaria, en la que se respeten los derechos y libertades fundamentales y de igualdad entre hombres y 
mujeres”. Esto ha llevado a que en los nuevos planes de estudio se promueva no sólo la consecución de competencias específicas científicas y técnicas relacionadas con los saberes disciplinares (conocimientos teóricos y prácticos necesarios para el ejercicio de la profesión), sino también de competencias genéricas y transversales, algunas de las cuales, de carácter interpersonal ${ }^{5}$, están orientadas al desarrollo de valores, comportamientos, actitudes y compromiso ético en los y las estudiantes.

En esta línea, la actual Ley Orgánica 1/2004, de 28 de diciembre, de Medidas de Protección Integral contra la Violencia de Género ${ }^{6}$, que se refiere a la violencia de género como un asunto que no es privado sino de todos, contempla medidas de sensibilización e intervención en el ámbito educativo, mencionando expresamente la necesidad de que las universidades incluyan y fomenten "en todos los ámbitos académicos la formación, docencia e investigación en igualdad de género y no discriminación de forma transversal” (art. 3.7). Asimismo, la vigente ley para la igualdad ${ }^{7}$ insta a las instituciones de enseñanza superior a promover la enseñanza y la investigación relativa a la igualdad entre mujeres y hombres (art. 25), destacando la corresponsabilidad y la prevención de la violencia como algunos de los ámbitos de gran interés para la promoción de nuevos valores habida cuenta de los obstáculos y estereotipos aún vigentes. Resultan sin embargo insuficientes los pasos dados en esa dirección, déficit que ha llevado a algunos autores a subrayar la urgencia en el "desarrollo de competencias para la responsabilidad compartida” (Civila, 2011, p. 11).

Con el fin de abordar estas cuestiones, desde la Universidad de Valladolid se coordinó durante el año 2013 un proyecto tendente a la promoción de la investigación y la formación desde el ámbito universitario de la corresponsabilidad como eje de democratización de las instituciones y de mejora de la calidad de vida de las personas. Dicho proyecto, denominado "Seminario iberoamericano de formación en corresponsabilidad", tenía entre sus principales objetivos el fomento de los valores de la corresponsabilidad y la no violencia entre la comunidad universitaria y las instituciones y entidades participantes y el impulso de la inclusión de estos temas en las materias troncales de los grados. El Seminario, que tenía un alcance internacional al participar en él departamentos de diversas universidades españolas y latinoamericanas ${ }^{8}$, integraba a docentes, alumnado y personal de administración y servicios, así como a personal técnico de diversas entidades colaboradoras. ${ }^{9}$ El Seminario se articuló en diversas fases que abarcaron desde la sensibilización y difusión de las temáticas objeto de estudio, hasta el diseño y la puesta en marcha de un curso de formación online, la organización de talleres y, para finalizar, el desarrollo de un encuentro iberoamericano para la puesta en común de aprendizajes y experiencias.

Retomando las cuestiones inicialmente mencionadas, y en el marco del SIFOC, se conformó -entre otros- un pequeño grupo de trabajo formado por PAS del servicio de bibliotecas y PDI para contribuir a idear una estrategia de trabajo colaborativo que, integrando a personal que habitualmente comparte espacios pero no proyectos ni objetivos en el cada vez más complejo marco organizativo de nuestros centros de enseñanza superior, permitiera aunar esfuerzos en la línea de promover hacia adentro formas diferentes de ser y de hacer y, hacia fuera, un modelo de universidad más comprometido con la transmisión de valores para una sociedad más justa, solidaria y equitativa.

\section{LA BIBLIOTECA UNIVERSITARIA Y LA CORRESPONSABILIDAD}

Las bibliotecas universitarias se han caracterizado por estar en continua evolución, alerta a los cambios en la sociedad y su repercusión en la institución que las alberga. Así pues, en la última década era de esperar el enorme salto que han aportado, a todos los niveles, las nuevas técnicas de información y comunicación (TICs) y la implantación en las universidades españolas del Espacio Europeo de Educación Superior (EEES), cuya consecuencia inmediata es un replanteamiento del concepto de biblioteca universitaria.

Durante las Jornadas REBIUN (Red de Bibliotecas Universitarias Españolas) celebradas en Palma de Mallorca en 2003, se expusieron diversas ideas y proyectos de lo que debe ser el nuevo modelo de biblioteca para servir, e incluso anticiparse, a las previsibles exigencias del nuevo modelo educativo. Todo ello se plasma en el famoso informe dirigido por Manuel Area (2004), donde se establecen las pautas para la gran transformación. El título del informe es significativo: "De la biblioteca universitaria al centro de recursos para el aprendizaje e investigación. Informe final” y se basa en un modelo británico, integrador de todos los recursos necesarios para el buen desarrollo de la docencia, la investigación y el aprendizaje, el Resource Learning Centre (RLC), que en España dio en llamarse CRAI (Centro de Recursos para el Aprendizaje y la Investigación).

Resumiendo mucho, los CRAI, en palabras de Moscoso (2003, p. 2), "responden a una estructura que integra servicios y recursos bibliotecarios, tecnológicos y audiovisuales; sistemas de información; e instalaciones y medios para la edición electrónica y la creación de materiales interactivos, a fin de dar el debido soporte a las necesidades 
docentes y de aprendizaje de la comunidad universitaria". Igualmente, Martínez (2004, p. 1), ofrece un detallado análisis sobre la organización de un centro de recursos y los pasos para convertir una biblioteca tradicional, "servicio clásico de la universidad, como depositaria de la producción científica y técnica de todos los conocimientos", hasta llegar a ser la "punta de la innovación en política de servicios universitarios". La biblioteca ha pasado pues a convertirse en un elemento estratégico y transversal de la universidad, donde confluyen todos los servicios que tienen "relación directa con el aprendizaje y el desarrollo personal de los miembros del campus".

El CRAI es un concepto espacial, en el sentido de que pretende hospedar, en un único emplazamiento (físico o virtual), una serie de servicios clave: acogida a nuevos estudiantes, soporte informático, laboratorio de idiomas, búsqueda de empleo, soporte y formación del profesorado, creación y recopilación de materiales docentes... y, por supuesto, servicio de biblioteca que, de hecho, será el núcleo central en torno al que giren el resto de servicios. El servicio de biblioteca, como indica Martínez (2004), es muy amplio y sus diversos apartados se podrían agrupar en tres grandes categorías según las necesidades de usuarios y usuarias: para el aprendizaje, para la investigación y servicios digitales. En la primera categoría podríamos ubicar todos los servicios relacionados con la formación en competencias informacionales y de orientación para el autoaprendizaje, objeto del presente trabajo.

Esta nueva concepción de la biblioteca universitaria implica un nuevo modelo del profesional de la información que, según López Yepes (2007, p. 268), va a estar sujeto a permanente renovación, va a verse inmerso en una comunidad científica más cosmopolita y compleja que le ofrece amplias posibilidades de colaboración y, sobre todo, que le empuja a ser "colaborador de la conversión de la información en conocimiento frente al mero papel de transmisor de la información indiscriminada” y que le invita a convertirse en activo "colaborador de la formación permanente de los ciudadanos desde la consideración de la educación como base de la nueva sociedad de la información”.

REBIUN ha trabajado intensamente en estos aspectos para orientar a las bibliotecas universitarias en la implantación de servicios de ALFIN (Alfabetización Informacional), cuyo resultado más visible es el portal web CI $2^{10}$, acrónimo de Competencias Informáticas e Informacionales, que emana de la CRUE-TIC (Comisión sectorial de Tecnologías de la Información y las Comunicaciones de la Conferencia de Rectores de Universidades Españolas). En este contexto, REBIUN se marca como objetivo "la incorporación de estas competencias transversales en las universidades españolas [...y ] la evolución necesaria para adaptar las competencias informacionales al ámbito digital”.

También en el seno de REBIUN van tomando cuerpo las ideas de colaboración, alianzas e integración de servicios universitarios, al tiempo que empieza a emerger la noción de Responsabilidad Social Universitaria (RSU). Así lo expone Marraud (2013) en las últimas “Jornadas españolas de documentación FESABID”, siguiendo la estela de Kagan (2005) en cuanto a la definición de responsabilidad social bibliotecaria y transmitiendo nociones similares a las que reflejan los programas desarrollados conjuntamente por ALA (Asociación Americana de Bibliotecas) e IFLA (Federación Internacional de Asociaciones de Bibliotecarios e Instituciones). Kagan (2005) recopila los temas que afloran de manera recurrente en dichos programas, entre ellos el feminismo y las cuestiones de género, junto con otros que reflejan la implicación social de la biblioteca en temas relacionados con el medio ambiente y la sostenibilidad, la desigualdad social, la libertad sexual, la independencia intelectual, etc. Más recientemente, el $80^{\circ}$ Congreso General de la IFLA ha tenido uno de sus momentos culminantes en la presentación de la Declaración de Lyon (2014) ${ }^{11}$ sobre el acceso a la información y el desarrollo, en la que destaca la importancia de los intermediarios de la información, como son bibliotecas y archivos, en el acceso, comprensión y utilización de la información para promover el desarrollo sostenible y las sociedades democráticas.

REBIUN, por su parte, ha plasmado estas tendencias en su Plan Estratégico $2020^{12}$ marcándose, entre otros, los siguientes objetivos:

“Línea 1. Objetivo 5: Promover la integración de la biblioteca en los objetivos de la universidad. Fortalecer alianzas con otros servicios universitarios e implicar las bibliotecas universitarias en el ámbito de la responsabilidad social de la universidad.

Línea 2. Epígrafe 3: Desarrollar y mejorar el modelo de biblioteca universitaria como Centro de Recursos de Aprendizajes e Investigación [...] estableciendo marcos de colaboración e integración con otros servicios universitarios".

La nueva definición de biblioteca universitaria acordada en la última Asamblea REBIUN ${ }^{13}$ (7 y 8 de noviembre de 2013) incide sobre estas mismas ideas:

"La biblioteca es un centro de recursos para el aprendizaje, la docencia y la investigación y las actividades relacionadas con el funcionamiento y la gestión de la universidad/institución en su conjunto. La biblioteca 
tiene como misión facilitar el acceso y la difusión de los recursos de información y colaborar en los procesos de creación del conocimiento a fin de contribuir a la consecución de los objetivos de la universidadinstitucionales, realizando las actividades que esto implica de forma sostenible y socialmente responsable”.

Este principio de colaboración, concretamente de colaboración de forma "socialmente responsable", ha dado sus frutos en diversas experiencias de formación en competencias informacionales que implican a PDI y personal bibliotecario y que han sido relatadas en algunos trabajos que citamos: Aguilera, Hernández-Pérez, Ramos y Vianello (2010); Santana Hidalgo, et al. (2011); Arévalo-Baeza, Domínguez-Aroca e Iglesia-Sánchez (2012). En ellos, se presenta a personal bibliotecario y docente trabajando codo con codo en la formación del alumnado para propiciar el autoaprendizaje y el aprendizaje a lo largo de la vida, según las pautas de Bolonia y el nuevo concepto de biblioteca universitaria que venimos analizando.

Asimismo, puede resultar muy inspiradora alguna iniciativa internacional de incluir la perspectiva de género en bibliotecas, especialmente la Guía editada por la Dirección de Bibliotecas, Archivos y Museos de Chile (Maillard, 2012), cuyo objetivo es sistematizar su experiencia así como otras iniciativas para la incorporación del enfoque de género en bibliotecas de manera que puedan convertirse en material de apoyo para nuevas acciones u otras similares.

Sin perder de vista las citadas fuentes, en el presente trabajo se presenta una experiencia que pretende ir un paso más adelante y enriquecer esta necesaria colaboración en dos vertientes:

- Por una parte, adoptando el término corresponsabilidad como un concepto más amplio y más comprometido que los ya citados (colaboración, alianzas, integración...). Corresponsabilidad entre PDI, alumnado y personal de la biblioteca para la formación en competencias informacionales pero también en otros valores sociales, éticos, humanos...

- La segunda vertiente parte de nuestro propio aprendizaje en el SIFOC, tanto en corresponsabilidad en las organizaciones -aquí, en el ámbito universitario- como en corresponsabilidad para la prevención de la violencia de género, para plantear actividades de alfabetización informacional que pueden ser el vehículo apropiado para la difusión de los valores de equidad y no discriminación por razones de género en la comunidad universitaria, especialmente entre el alumnado.

Estos presupuestos teóricos se han plasmado en tres acciones formativas, elaboradas desde las bibliotecas de Económicas y Filosofía y Letras de la UVa, que se detallan en los siguientes epígrafes.

\section{UNA PROPUESTA FORMATIVA PARA LA PROMOCIÓN DE LA CORRESPONSABILIDAD Y LA PREVENCIÓN DE LA VIOLENCIA DE GÉNERO}

La vigente normativa española, tal y como se ha señalado, exige promover valores como la igualdad de oportunidades entre mujeres y hombres en los planes de estudios de los nuevos grados, pero también incide en la necesidad de crear redes cooperativas de conocimiento, según los presupuestos del EEES. La biblioteca, como servicio universitario de carácter transversal, puede enriquecer estas redes de aprendizaje y aportar sus conocimientos y experiencia en gestión bibliográfica y formación en competencias informacionales y transversales.

Como ya se ha comentado, a lo largo de 2013 parte del personal de las bibliotecas de Ciencias Económicas y de Filosofía y Letras de la UVa participó en el SIFOC y, durante su desarrollo, pronto se hizo evidente que la biblioteca, además de colaborar y asesorar en la búsqueda y uso de información, también podía asumir un papel activo en el diseño y puesta en marcha de talleres formativos para la prevención de la violencia de género y el fomento de valores conducentes a una sociedad más justa y democrática.

La participación del personal de biblioteca en el SIFOC se concretó por tanto no sólo en la asistencia a sus reuniones, aportando ideas y sugerencias desde el punto de vista de su condición de personal especializado en cuestiones de documentación, participando en grupos de trabajo encargados de desarrollar actividades concretas (presentación de libros, observatorio de buenas prácticas, etc.) sino que, a partir de la realización de un curso de formación en materia de corresponsabilidad como estrategia para la prevención de la violencia de género, se dio el paso de que desde la biblioteca se contribuyera al diseño de acciones formativas tendentes a sensibilizar y formar en dichas materias. Se contaba además para ello con una experiencia de años en el manejo de numerosos y diversos recursos de información, impresos o electrónicos, adquiridos por la Biblioteca de la Universidad de Valladolid (BUVa) o externos a ella; en la selección, evaluación y comunicación responsable de dicha información, así como en la formación general o especializada sobre la biblioteca, sus recursos y servicios. Sin olvidar que la Biblioteca cuenta con personal, instalaciones y medios tecnológicos adecuados para impartir cursos y talleres. 
La Biblioteca, alineada con los principios de la institución, desarrolla y ofrece algunos servicios cuya filosofía podría enmarcarse en el concepto de corresponsabilidad entre integrantes de la comunidad universitaria. En este sentido, el personal de la BUVa integrante del Seminario coordina o participa en varios servicios bibliotecarios, en estrecha colaboración con PDI, alumnado y personal informático de la Universidad, como son:

1. Servicio La Biblioteca con la Investigación ${ }^{14}$, que implica un compromiso de la Biblioteca con el PDI a la hora de asesorarle y apoyarle en temas relacionados con la redacción y publicación de trabajos, acreditaciones, solicitud de sexenios de investigación, etc.

2. Portal institucional de la UVa y Directorio de Autores ${ }^{15}$ en Dialnet: la Biblioteca trabaja en este portal para la identificación, actualización y visibilidad del PDI de la UVa, de sus publicaciones y de la producción científica generada en nuestra Universidad.

3. Repositorio institucional UVaDOC ${ }^{16}$ : la Biblioteca coopera con PDI y alumnado para ofrecer en acceso abierto tesis doctorales, trabajos de fin de grado y trabajos de fin de máster, así como objetos de aprendizaje, artículos, contribuciones a congresos, etc.

4. Alfabetización informacional: a la formación presencial para estudiantes de grado y máster, organizada siempre en colaboración con el PDI, hay que sumar dos nuevos proyectos: un curso online sobre recursos especializados en economía y empresa y otro, organizado con otras bibliotecas y servicios bibliotecarios de la UVa, dirigido a estudiantes de doctorado, con el apoyo de la Escuela de Doctorado y del personal informático.

5. Difusión selectiva de la información a través de múltiples medios de comunicación, con especial atención a las redes sociales: blog BibEcoUva ${ }^{17}$, Guía de Recursos Especializados ${ }^{18}$ (en Delicious), etc.

En consecuencia, los talleres de formación para la prevención de la violencia de género que presentamos sintéticamente a continuación surgen a raíz de la participación del personal de las bibliotecas de la Facultad de Ciencias Económicas y de Filosofía y Letras en el SIFOC, pero también como resultado lógico de su experiencia bibliotecaria y su implicación con la institución a la que pertenecen. Esta propuesta formativa tiene el valor de haber sido el fruto, a su vez, de un proceso de formación en materia de igualdad y corresponsabilidad que se desarrolló en el marco del citado proyecto y en el que participó -entre otros destinatarios- personal del servicio de bibliotecas, y de haber sido analizada y evaluada positivamente por las personas expertas en temas de género que impartieron dicho curso de formación. Entendemos que dicha experiencia es en sí de enorme interés, no sólo porque permite divulgar el potencial que tiene la colaboración entre el personal de bibliotecas y otros colectivos dentro y fuera del ámbito universitario para reforzar los compromisos en materia de responsabilidad social que tienen las instituciones universitarias, sino también porque pone a disposición de otras personas interesadas los procedimientos y herramientas necesarios para replicar la iniciativa adaptándola a las peculiaridades de otros centros, servicios, etc.

\subsection{Taller para la detección, análisis y prevención del sexismo en la publicidad}

El primer taller tiene como objetivo la detección, análisis y prevención de la publicidad sexista en los medios de comunicación y está destinado a estudiantes de enseñanza superior, futuros especialistas en marketing, investigación de mercados y publicidad.

Quien esté más o menos sensibilizado hacia la desigualdad de género en la sociedad occidental, no ha podido dejar de percibir que la publicidad actual está plagada de modelos y estereotipos sexistas que perpetúan la idea de la mujer como ser inferior, dependiente o mero objeto de deseo sexual. Dicha visión distorsionada del papel de la mujer en la sociedad puede considerarse como una forma sutil de violencia de género y contribuye sin duda a la perpetuación de la desigualdad (León, 1996). Si bien algunos estudios recientes constatan un "menor uso publicitario de roles tradicionales para ambos géneros” (Royo et al., 2005, p. 132), la mayoría de los trabajos subrayan la persistencia de prejuicios que impiden la igualdad real (Espín, 2002).

Sin embargo, no vale la sola intuición y se hace necesario el análisis detallado y la búsqueda de alternativas para concienciar a quienes en el futuro se encargarán de difundir imágenes de la necesidad de ser más ecuánimes y de disponer de una conciencia colectiva basada en la corresponsabilidad, en la que estén erradicadas las desigualdades. La educación en estos valores parece ser la garantía ${ }^{19}$ para conseguir una sociedad más equitativa y ética, donde no tenga cabida la violencia de género.

La transversalidad de la biblioteca, como servicio universitario que atañe a todos los colectivos de la institución, vendría a enriquecer estas redes de aprendizaje cooperativo, aportando sus conocimientos y experiencia en cuanto a gestión bibliográfica y formación de usuarios en cuanto a identificación de sus necesidades de información, localización y utilización ética y responsable de dicha información. Así, el taller se convierte en una red de creación de conocimientos basado en la corresponsabilidad entre docentes, estudiantes y personal bibliotecario. 


\section{TALLER PARA LA DETECCIÓN, ANÁLISIS Y PREVENCIÓN DEL SEXISMO EN LA PUBLICIDAD}

Destinatarios/as: Alumnado de Grado de Marketing e Investigación de Mercados de la Uva.

$\mathbf{N}^{0}$ aconsejable de plazas: 15 a 20 asistentes.

\section{Objetivos:}

1. Concienciar y sensibilizar sobre la existencia y persistencia de muchos aspectos sexistas en la publicidad difundida a través de los medios de comunicación audiovisual (prensa, radio, televisión, internet).

2. Capacitar para detectar el sesgo sexista en anuncios publicitarios y plantear alternativas no sexistas basadas en la equidad y corresponsabilidad y orientadas a la prevención de la violencia de género.

\section{Metodología y cronograma:}

Taller semipresencial de $20 \mathrm{~h}$ de duración, con $7 \mathrm{~h}$ presenciales (resto de trabajo personal o en grupo) repartidas en tres sesiones a lo largo de tres semanas:

- Sesión introductoria: Presentación del taller, formación de grupos de trabajo (preferentemente mixtos, de 3-4 personas), asignación de un tema de trabajo (propuestos en "Contenidos”), elección del modelo y formato de trabajo. Entrega de la guía didáctica y del material bibliográfico y documental. Nociones sobre técnicas de búsqueda bibliográfica. Creación de un foro y una lista de distribución de correo electrónico para interactuar. Semana 1. $2 \mathrm{~h}$.

- Sesión de seguimiento de los trabajos: el alumnado presenta el tema y estructura de su trabajo. Resolución de dudas y profundización en estrategias de búsqueda y tratamiento de la información. Semana 2. 2h.

- Sesión final de presentación de los trabajos: cada trabajo será presentado en una exposición pública (15 minutos) seguida de un breve debate entre asistentes y coordinadores. Semana 3. 3h.

\section{Contenidos:}

Partiendo de una visión sexista y de perpetuación de estereotipos de género que presenta la publicidad actual en diferentes ámbitos (pareja, familia, hogar, trabajo fuera de casa, ocio, imagen propia o ajena...), el taller busca incidir en alternativas para mostrar una imagen de equidad y corresponsabilidad entre géneros. Cada grupo tratará en su trabajo uno de los siguientes temas, extraídos de alguno de los estereotipos sexuales más extendidos en publicidad:

- $\quad$ Papeles tradicionalmente asignados a la mujer: hogar y familia, ajena al mundo profesional;

- $\quad$ mujer dependiente, pasiva y sumisa respecto al hombre;

- $\quad$ consecución del éxito social basada en determinado canon de belleza femenina;

- $\quad$ cuerpo femenino como reclamo del cliente (masculino o femenino);

- $\quad$ tópicos despectivos acerca de las mujeres (caprichosas, histéricas, charlatanas...).

\section{Recursos didácticos:}

- Recursos humanos: colaboración entre docentes y el personal bibliotecario del centro. El profesorado se responsabilizará del diseño, seguimiento y evaluación final del taller (como práctica de la asignatura). El personal bibliotecario aportará asesoramiento bibliográfico y de formación en competencias informacionales.

- Recursos materiales y tecnológicos: aula multimedia versátil, dotada de medios tecnológicos y audiovisuales, que favorezca la interactuación entre los y las participantes.

- Recursos bibliográficos y de información: el profesorado y la biblioteca proporcionarán la bibliografía necesaria y pertinente y la orientación para conseguir información complementaria. 


\section{Evaluación:}

Para la evaluación de los trabajos, una vez expuestos públicamente, se plantean dos métodos sincrónicos:

- Valoración por parte de los compañeros y compañeras en el debate final.

- Valoración del profesorado, que tendrá en cuenta la coherencia global del trabajo y criterios como el número y variedad de anuncios analizados, la adecuación del material presentado al tema seleccionado, la presentación del trabajo (claridad expositiva, corrección lingüística y uso de lenguaje no sexista), el empleo de diferentes medios de análisis (textuales o audiovisuales), la sensibilidad y creatividad en las alternativas no sexistas aportadas.

Se evaluará también el desarrollo del propio taller mediante encuestas de satisfacción de participantes.

\section{Bibliografía básica:}

- ESPÍN, J.V.; GRACIA, M.A. y LAJO, M.R. Las imágenes de las mujeres en la publicidad: estereotipos y sesgos [en línea]. Redes.com: revista de estudios para el desarrollo social de la comunicación, 2006, $\mathrm{n}^{\circ}$ 3, p. 77-90. ISSN: 1696-2079. Disponible en: <http://dialnet.unirioja.es/servlet/articulo?codigo=3664567> [Consulta: 26 de septiembre de 2014].

- GARCÍA, M.A.C. La imagen de la mujer en los medios de comunicación: remedios contra la publicidad sexista. En: G. Fabregat Monfort (Ed.). Mujer y derecho: Jornada de igualdad de la Facultad de Derecho de la Universidad de Valencia. Valencia: Tirant lo Blanch, 2011, p. 195-218.

- HERNÁNDEZ RUIZ, A.; MARTÍN LLAGUNO, M. y BELÉNDEZ VÁZQUEZ, M. La representación de la mujer en publicidad: (des) igualdad cuantitativa y cualitativa en la creatividad española [en línea]. Estudios sobre el Mensaje Periodístico, 2012, no 18 (nº especial), p. 521-530. ISSN 1134-1629. Disponible en: <http://revistas.ucm.es/index.php/ESMP/article/view/40931> [Consulta: 26 de septiembre de 2014].

- HERNÁNDEZ RUIZ, A. y MARTÍN LLAGUNO, M. El sexismo en publicidad: estructuras, discursos y recomendaciones [en línea]. Historia y comunicación social, 2013, vol. 18, nº 1, p. 147-156. ISSN-e: 19883056. Disponible en: <http://revistas.ucm.es/index.php/HICS/article/view/43955> [Consulta: 26 de septiembre de 2014].

Tabla I. Taller para la detección, análisis y prevención del sexismo en la publicidad.

\subsection{Taller de formación para la detección y prevención de la violencia de género dirigido a estudiantes de periodismo $^{20}$}

El uso de estereotipos de género constituye una práctica discriminatoria y, en ocasiones, germen de violencia en distintos grados o manifestaciones. Los estereotipos en ocasiones están vinculados a una serie de circunstancias sociales y culturales, y son los propios profesionales del periodismo los que los usan con frecuencia de manera inconsciente por falta de atención, de espíritu crítico y/o de sensibilidad ante lo que representan. Ciertamente ya no son hoy frecuentes los abordajes explícitamente sexistas de antaño en la información periodística, pero sí persisten formas discriminatorias sutiles y muy efectivas de tratamiento "tanto en los tiempos y espacios dedicados a la mujer por los medios como en su forma cualitativa de concreción” (Torregrosa, 2008, p. 138).

Los y las periodistas, como comunicadores sociales que son, tienen la gran responsabilidad de analizar y describir la realidad no sólo evitando los mensajes discriminatorios hacia las mujeres, sino intentando contribuir a la reducción de las desigualdades de género (Chaher y Santoro, 2007). Este no es un tema que concierna sólo a las mujeres, sino que es un asunto de interés y bienestar común y, por tanto, necesario e imprescindible para el desarrollo global de una sociedad plenamente democrática.

En el entorno educativo en el que nos encontramos, la UVa imparte el Grado de Periodismo y es responsable de la formación y capacitación de futuros profesionales de la comunicación. Asimismo, esta Universidad está comprometida con la equidad de género y así lo ha hecho explícito a través del Plan de Igualdad entre Hombres y Mujeres de la UVa 2012-2014. ${ }^{21}$

Así pues, existe el marco normativo, el ámbito profesional clave en la comunicación, y la voluntad de sensibilización y concienciación idóneos para el desarrollo de una taller formativo transversal y cooperativo en el que participen docentes, estudiantes y la biblioteca universitaria para formar futuros y futuras profesionales en periodismo que contribuyan conscientemente a la equidad de género y al afianzamiento de un sistema democrático basado en la justicia social. 


\section{TALLER DE FORMACIÓN PARA LA DETECCIÓN Y PREVENCIÓN DE LA VIOLENCIA DE GÉNERO DIRIGIDO A ESTUDIANTES DE PERIODISMO}

Destinatarios/as: Alumnado del Grado de Periodismo de la UVa.

$\mathbf{N}^{\circ}$ aconsejable de plazas: 15 a 20 asistentes.

\section{Objetivos:}

1. Crear un contexto de sensibilización hacia el sexismo presente en los medios de comunicación, tanto en prensa escrita como en radio, televisión y otros medios de comunicación social.

2. Adiestrar en la detección de situaciones, expresiones y contextos relacionados con la violencia de género y, por tanto, contrarios a la neutralidad que debe tener una noticia periodística.

3. Fomentar el espíritu crítico y que el alumnado sea capaz de elaborar propuestas correctoras de las noticias como paso necesario para la formación en la prevención de la violencia de género.

4. Que el alumnado adquiera una capacidad consciente de comunicación libre de sexismo que desarrollen a lo largo de su vida personal y profesional, convirtiéndose así en futuros profesionales de la comunicación comprometidos con una sociedad plenamente democrática.

\section{Metodología y cronograma:}

Taller semi-presencial de 20h de duración, con $7 \mathrm{~h}$ presenciales repartidas a lo largo de 5 semanas:

- Sesión introductoria: Introducción teórica donde se plantearán los contenidos básicos, se proporcionará la bibliografía y documentación y se constituirán los grupos de trabajo. Presentación de los/las estudiantes y creación de grupos de trabajo (mixtos y 3 o 4 personas máximo). A cada grupo se le asignará un trabajo diferente cuyo objeto será el análisis y evaluación de noticias publicadas o emitidas en medios de comunicación distintos y/o en diferentes contextos vitales en los que haya situaciones de violencia de género. Semana 1. $2 \mathrm{~h}$.

- Sesión de seguimiento: cada grupo expondrá el trabajo a desarrollar, motivando brevemente su elección en términos de prevención de violencia, sexismo, equidad de género, y corresponsabilidad. En esta sesión se reforzarán conceptos clave, se plantearán dudas, etc. Semana 2. 2h.

- Foro de participación y debate. Junto al trabajo personal y grupal, y para el planteamiento de dudas o consultas, debate de propuestas y sugerencias, planteamiento de nuevas noticias para conocimiento general, etc., los grupos de contarán con un foro virtual de debate. Semanas 3 y 4.

- Sesión final. Todos los trabajos realizados serán expuestos públicamente, ya sea por medio de informes analíticos, grabaciones de vídeo o podcast, redacción de noticias nuevas, elaboración de entrevistas, etc., y se procederá a su evaluación. Semana 5. 3h.

\section{Contenidos:}

Se analizarán noticias publicadas en prensa donde se detecte sexismo y desencadenen situaciones de violencia de género, haciendo especial hincapié en contextos de la vida ordinaria dado que éstos son los que más fácilmente pasan desapercibidos.

Las noticias pueden extraerse de cualquier medio de comunicación pública, preferiblemente en prensa, radio y televisión de difusión nacional, sin excluir medios locales si la noticia tiene proyección suficiente. Se hará hincapié en los siguientes aspectos:

- $\quad$ Análisis de ideas preconcebidas y estereotipos sexistas;

- manifestación y denuncia de actos que no respetan a la mujer, sus derechos, ideas, capacidades, decisiones, situación, trabajo, libertad sexual, etc.;

- $\quad$ situaciones de violencia de género en todas sus manifestaciones. 


\section{Recursos didácticos:}

Se proporcionará al alumnado herramientas de apoyo: bibliografía y bases de datos con información sobre prevención de la violencia y sobre equidad de género, webs de periodistas corresponsables en temas de igualdad y concienciación democrática, ejemplos de buenas prácticas en comunicación, formación en valores y ética periodística, etc. Aunque se proporcionará material bibliográfico y documental suficiente, se fomentará y valorará que los y las estudiantes busquen otra información pertinente a través de los recursos y servicios que proporciona la Biblioteca.

Se reservará un Aula Multimedia, dotada de equipos informáticos, de audio y video.

\section{Evaluación:}

Habrá dos evaluaciones de las actividades desarrolladas por el alumnado: $1^{\circ}$ cada grupo será evaluado por sus propios compañeros/as creando así un entorno de diálogo constructivo y de retroalimentación; y $2^{\circ}$ el profesorado valorará al alumnado en relación con el trabajo realizado y los objetivos planteados.

Finalmente, el alumnado cumplimentará una encuesta de evaluación del taller y del profesorado.

\section{Bibliografía básica:}

- COMITÉ DIRECTIVO PARA LA IGUALDAD ENTRE HOMBRES Y MUJERES (CONSEJO DE EUROPA). Manual sobre los estereotipos de género en los medios de comunicación [en línea], (s.f.). Disponible en: <http://www.inmujer.gob.es/servRecursos/portada/docs/manual.pdf> [Consulta: 26 de septiembre de 2014].

- CHAHER, S. y SANTORO, S. (Comp.). Las palabras tienen sexo. Introducción a un periodismo con perspectiva de género [en línea]. Buenos Aires, Artemisa Comunicación Ediciones, 2007. ISBN: 978-98723611-0-5. Disponible en: <http://www.artemisanoticias.com.ar/images/las-palabras-tienen-sexo.pdf> [Consulta: 26 de septiembre de 2014].

- CHAHER, S. y SANTORO, S. (Comp.). Las palabras tienen sexo II. Herramientas para un periodismo de género. Buenos Aires, Artemisa Comunicación Ediciones, 2010. ISBN: 978-987-23611-1-2

- RODRÍGUEZ, M.P. Periodismo de opinión: hacia las buenas prácticas en el tratamiento de la violencia de género. En: Tratamiento de la violencia de género en la prensa vasca. San Sebastián, Universidad de Deusto, 2006, p. 115-150. ISBN 84-9830-040-1

- RODRÍGUEZ CÁRCELA, R. Del crimen pasional a la violencia de género: evolución y su tratamiento periodístico [en línea]. Ámbitos: Revista internacional de comunicación, 2008, nº 17, p. 171-188. ISSN-e 1139-1979. Disponible en: <http://grupo.us.es/grehcco/ambitos17/11Rodriguez.pdf> [Consulta: 26 de septiembre de 2014].

Tabla II. Taller de formación para la detección y prevención de la violencia de género dirigido a estudiantes de periodismo.

\subsection{Taller de formación en Dialnet para la corresponsabilidad y la prevención de la violencia de género.}

Este taller surgió a raíz de la participación y cooperación con otro de los grupos de trabajo formados dentro del SIFOC -el Observatorio SIFOC-, cuyo objetivo era la investigación y análisis de iniciativas de buenas prácticas en corresponsabilidad. Fruto de esta colaboración fue la creación en Dialnet de dos listas bibliográficas de documentos seleccionados sobre los temas objeto del Seminario. Ambas listas se actualizan permanentemente y se han abierto al público para que puedan ser consultadas, utilizadas y difundidas por cualquier persona interesada, pertenezca o no a la UVa, desde cualquier parte del mundo. Son las siguientes:

- Bibliografía sobre Corresponsabilidad familiar ${ }^{22}$

- Bibliografía sobre Prevención de la violencia de género en todos los ámbitos ${ }^{23}$

Dialnet es un proyecto en el que actualmente colaboran 60 bibliotecas universitarias -53 españolas y 7 latinoamericanas-, 16 bibliotecas especializadas y 4 bibliotecas públicas -entre ellas la Biblioteca Nacional de España-, que son las que alimentan sus bases de datos de revistas, tesis, congresos, libros y capítulos de libros, etc.

La Biblioteca de la UVa colabora con Dialnet desde 2006 ocupándose de un centenar de revistas, incluidas las editadas por la propia institución, así como de libros colectivos y publicaciones de congresos en los que participan miembros de nuestra comunidad universitaria. El objetivo perseguido es proporcionar servicios de información y, sobre todo, fomentar la visibilidad de la producción científica e investigadora de la UVa.

Dialnet es hoy día uno de los principales recursos de información en español, y, en consecuencia, es reconocido por el Ranking Web de Repositorios del Mundo ${ }^{24}$ elaborado por el Laboratorio de Cibermetría del CSIC como el 
segundo portal de información del mundo en cuanto a contenido bibliográfico y documental. ${ }^{25}$ En este contexto, Dialnet constituye una fuente de información fundamental tanto para buscar y localizar referencias y documentos sobre la violencia de género y su prevención, como para difundir otros proyectos e iniciativas desarrolladas por cualquier persona que investigue, estudie o se interese en estos temas.

\begin{tabular}{|l}
\hline TALLER DE FORMACIÓN EN DIALNET PARA LA CORRESPONSABILIDAD Y LA \\
PREVENCIÓN DE LA VIOLENCIA DE GÉNERO \\
Destinatarios/as: alumnado de cualquier Grado, Máster o titulación impartida en la UVa, así como PDI y \\
PAS interesados en recursos de información en español (Dialnet) y la problemática de la violencia de género.
\end{tabular}

\section{Objetivos:}

1. Adiestrar en la búsqueda de información y enseñar a utilizar los servicios avanzados de Dialnet Plus a los que tiene acceso la comunidad universitaria de la UVa.

2. Concienciar y sensibilizar a la comunidad universitaria en materias relacionadas con la violencia de género y su prevención, el sexismo y la corresponsabilidad en sentido amplio.

3. Fomentar el espíritu crítico y los valores sociales como paso necesario para la formación de las personas, y por extensión de la sociedad, en la prevención de la violencia de género.

\section{Metodología y cronograma:}

El taller se impartirá de forma presencial, mediante la exposición de los contenidos y la realización de prácticas específicas en la búsqueda, gestión y utilización de documentos sobre violencia de género, prevención, sexismo y corresponsabilidad.

El taller se desarrollará en tres horas, destinándose el 50\% de tiempo a impartir los contenidos teóricos y el otro $50 \%$ al desarrollo de las prácticas y resolución de dudas.

\section{Contenidos:}

El taller consistirá en buscar en Dialnet información sobre violencia de género, prevención, sexismo, corresponsabilidad familiar, etc. Se contemplarán los siguientes aspectos:

1. Contenido de Dialnet, catálogo de revistas y gestión de alertas;

2. Dialnet Plus y servicios avanzados para la comunidad universitaria de la Uva;

3. búsqueda de información: buscador avanzado y posibilidad de guardar búsquedas favoritas;

4. localización y petición de documentos a la biblioteca a través de Dialnet;

5. gestión y utilización de los resultados de las búsquedas: listas de bibliografía, y exportación de resultados a gestores de referencias (Refworks) y gestores sociales (Mendeley).

\section{Recursos didácticos:}

El recurso principal es Dialnet Plus, cuyos servicios avanzados están destinados únicamente a miembros de las instituciones colaboradoras.

\section{Evaluación:}

Encuesta suministrada al alumnado donde evaluarán al profesorado, la metodología y los contenidos. Dicha encuesta recogerá también las opiniones de los y las asistentes y sus sugerencias, elementos fundamentales de retroalimentación y revisión del taller.

\section{Bibliografía:}

Listas bibliográficas creadas en Dialnet sobre corresponsabilidad <http://dialnet.unirioja.es/ref/4bl6> y sobre prevención de la violencia de género <http://dialnet.unirioja.es/ref/x96N $>$, que constituyen tanto una fuente de información en sí mismas, como un ejemplo de la utilidad de Dialnet a la hora de difundir información.

Tabla III. Taller de formación en Dialnet para la corresponsabilidad y la prevención de la violencia de género.

\section{CONCLUSIONES}

Hoy más que nunca necesitamos organizaciones corresponsables donde todos los miembros estén comprometidos con la tarea de formar ciudadanos y ciudadanas competentes pero también implicados en la promoción del bienestar, 
la equidad y el desarrollo social. En este sentido, es fundamental promover en nuestras instituciones de enseñanza superior las alianzas entre todos los colectivos universitarios, favorecer las interacciones entre las áreas de conocimiento, propiciar complicidades para potenciar nuevas formas más colaborativas de trabajar y potenciar con ello la contribución del sistema educativo a la creación de una sociedad mejor.

Bajo estas premisas, el proyecto SIFOC surge por y para comprender y difundir la idea de corresponsabilidad en el ámbito de las instituciones universitarias, implicando para ello de manera colaborativa a PDI, PAS y estudiantes en el aprendizaje de valores éticos y sociales como la corresponsabilidad como medio para prevenir la violencia de género en el seno de la propia organización y, por ende, en la sociedad en general.

La implicación del personal bibliotecario en el proyecto, en apariencia ajeno a su quehacer cotidiano, supone, en primer lugar, la toma de conciencia y sensibilización hacia aspectos sociales y de igualdad en el ámbito familiar y laboral, así como la comprensión de la estrecha relación entre corresponsabilidad y responsabilidad social corporativa, idea que está adquiriendo una enorme relevancia en el ámbito bibliotecario, como demuestran la última definición de biblioteca universitaria adoptada por REBIUN o la más reciente Declaración de Lyon sobre el acceso a la información y el desarrollo, firmada por más de 300 bibliotecas, organismos, asociaciones e instituciones internacionales.

En segundo lugar, entendemos que la posición estratégica de la biblioteca en la universidad, equidistante entre docencia, aprendizaje, investigación y gestión, brinda un inmejorable entorno de interrelación entre los diferentes colectivos universitarios. Espacios, recursos y servicios bibliotecarios, junto con los conocimientos y experiencia de su personal, pueden ser un vehículo idóneo para potenciar el compromiso social en la comunidad universitaria. Así pues, las bibliotecas, igual que otros servicios universitarios y en estrecha colaboración con ellos, pueden y deben aceptar los retos y compromisos que en materia de igualdad y equidad entre hombres y mujeres emanan de la LOMLOU, de la Ley de Medidas de Protección Integral contra la Violencia de Género y de la ley para la igualdad.

La experiencia muestra cómo la biblioteca universitaria puede ir más allá en sus funciones tradicionales y fortalecer su proyección social convirtiéndose no sólo en espacio para la generación activa y participada de materiales orientados a la adquisición y mejora de competencias informativas, sino también favoreciendo la promoción de valores sociales como la corresponsabilidad y la no violencia.

Lo expuesto hasta ahora, tanto en lo referente a la participación del personal de la biblioteca en un proyecto de docencia e investigación (SIFOC) como en el diseño de actividades de sensibilización y formación, puede ser perfectamente extrapolable a otros servicios bibliotecarios o universitarios y, a su vez, extensible a otros campos o temáticas que susciten en sus participantes el interés y el compromiso en temas sociales tales como los derechos humanos, la sostenibilidad, la justicia social, etc.

En definitiva, la experiencia descrita viene a confirmar tres cuestiones fundamentales: (a) la importancia del trabajo colaborativo entre los diferentes colectivos de la comunidad universitaria, (b) el nuevo y activo papel que puede jugar el servicio de biblioteca en todas las actividades propias de las organizaciones de enseñanza superior enseñanza, aprendizaje, investigación e innovación- y (c) la necesidad de impulsar nuevas acciones que estimulen y potencien no solo la sensibilización en materia de prevención de la violencia de género, sino también el compromiso social y el desarrollo de valores humanos en el seno de la comunidad universitaria.

\section{NOTAS}

\footnotetext{
${ }^{1}$ La necesidad de "repensar y construir un nuevo modelo de relaciones de corresponsabilidad" entre hombres y mujeres atañe por supuesto a muchos otros ámbitos y profesionales (Elósegui, 2009, p. 21).

${ }^{2}$ Proyecto interinstitucional e interuniversitario coordinado desde la UVa y financiado por el Instituto de la Mujer (Ministerio de Sanidad, Servicios Sociales e Igualdad) y el Fondo Social Europeo en el marco de la convocatoria de subvenciones destinadas a la realización de postgrados oficiales de igualdad entre mujeres y hombres, acciones complementarias a la investigación en el ámbito universitario y actividades de las unidades de igualdad de las universidades para el año 2012 (resolución de 14 de diciembre de 2012 del Instituto de la Mujer). Agradecemos al SIFOC la posibilidad que nos ha brindado de profundizar en la temática de la corresponsabilidad y la violencia de género y de llevar a cabo una experiencia colaborativa de interés y gran utilidad en nuestro propio contexto organizativo.

${ }^{3}$ Con la Comunicación de la Comisión Europea (2003) se abre el debate acerca de cómo conseguir estos objetivos en el marco de la Unión Europea ampliada.

4 ESPAÑA. 2007. Ley Orgánica 4/2007, de 12 de abril, por la que se modifica la Ley Orgánica 6/2001, de 21 de diciembre, de Universidades (LOMLOU). Boletín Oficial del Estado [en línea], 13 de abril de 2007, $\mathrm{n}^{\circ}$ 89, p. 16241-16260. Disponible en: $<$ http://www.boe.es/buscar/doc.php?id=BOE-A-2007-7786> [Consulta: 22 de septiembre de 2014].

${ }^{5}$ Siguiendo la tipología de competencias del Proyecto Tuning (González, J. y Wagenaar, R, eds. 2003).
} 
${ }^{6}$ ESPAÑA. 2004. Ley Orgánica 1/2004, de 28 de diciembre, de Medidas de Protección Integral contra la Violencia de Género. Boletín Oficial del Estado [en línea], 29 de diciembre de 2004, no 313, p. 42166-42197. Disponible en: <http://www.boe.es/boe/dias/2004/12/29/pdfs/A4216642197.pdf> [Consulta: 22 de septiembre de 2014].

${ }^{7}$ ESPAÑA. 2007. Ley Orgánica 3/2007, de 22 de marzo, para la Igualdad Efectiva entre Mujeres y Hombres. Boletín Oficial del Estado [en línea], 23 de marzo de 2007, $\mathrm{n}^{\circ}$ 71, p. 12611-12645. Disponible en: <http://www.boe.es/buscar/doc.php?id=BOE-A-2007-6115> [Consulta: 22 de septiembre de 2014].

${ }^{8}$ El personal universitario participante pertenecía a las universidades de: Valladolid, Complutense de Madrid, Islas Baleares, Autónoma Nacional de México (Sede Iztacala), Simón Bolívar de Barranquilla (Colombia).

${ }^{9}$ Asociación de Mujeres La Rondilla (Valladolid), Casa de la Mujer de Bolivia (Santa Cruz de la Sierra, Bolivia) y Fundación CEDID (Centro de Estudios Doctorales en Interculturalidad y Desarrollo. Santa Cruz de la Sierra, Bolivia).

${ }^{10}$ CI2 Competencias Informáticas e Informacionales [en línea]. Disponible en: <http://www.ci2.es/> [Consulta: 22 de septiembre de 2014].

11 The Lyon Declaration: on access to information and development [en línea]. 2014. Disponible en: < http://www.lyondeclaration.org/> [Consulta: 22 de septiembre de 2014].

12 REBIUN. III Plan Estratégico de REBIUN $2020 \quad$ [en línea]. <http://www.rebiun.org/queesrebiun/Documents/III_Plan\%20Estrategico_REBIUN.pdf> [Consulta: 22 de septiembre de 2014].

13 REBIUN. Asamblea anual (21 ${ }^{\text {a }}$ 2013. Zaragoza). Declaración y conclusiones de la Asamblea Anual de REBIUN [en línea]. Disponible en: $<$ http://www.comunidadbaratz.com/blog/declaracion-y-conclusiones-de-la-asamblea-anual-de-rebiun> [Consulta: 22 de septiembre de 2014].

14 UNIVERSIDAD DE VALLADOLID. La Biblioteca con la Investigación [en línea]. Disponible en: <http://biblioteca.uva.es/export/sites/biblioteca/3.servicios/3.09.labibliotecaconlainvestigacion/3.09.00.presentacion/index.html> [Consulta: 22 de septiembre de 2014].

${ }^{15}$ Portal institucional de la Universidad de Valladolid en Dialnet [en línea]. Disponible en: <http://dialnet.unirioja.es/institucion/buva> [Consulta: 22 de septiembre de 2014].

16 UVaDOC, Repositorio Documental de la Universidad de Valladolid [en línea]. Disponible en: <https://uvadoc.uva.es/> [Consulta: 22 de septiembre de 2014]

17 BIBECOUVA. El Blog de la Biblioteca de la Facultad de Ciencias Económicas de la Universidad de Valladolid [en línea]. Disponible en: $<$ http://bibecouva.blogs.uva.es/> [Consulta: 22 de septiembre de 2014].

${ }^{18}$ BibecoUva en Delicious [en línea]. Disponible en: <https://delicious.com/BibecoUva> [Consulta: 22 de septiembre de 2014].

19 García y Lema (2008) analizan diversas medidas posibles de intervención ante la publicidad sexista, entre las que cabe mencionar las de naturaleza jurídica. Los resultados de algunas de estas medidas, no obstante, parecen no resultar aún suficientemente eficaces (Navarro y Martín, 2012).

${ }^{20}$ Durante el año 2013, una de las coautoras estuvo trabajando en la Biblioteca de la Facultad de Filosofía y Letras, centro en el que se imparte el Grado de Periodismo donde se enmarca este taller.

${ }^{21}$ UNIVERSIDAD DE VALLADOLID. Plan de Igualdad de Oportunidades entre Mujeres y Hombres, 2012-2014 [en línea]. Disponible en: $<$ http://www5.uva.es/catedraestudiosgenero/Plan_Igualdad_UVa.pdf> [Consulta: 22 de septiembre de 2014].

22 Bibliografía sobre corresponsabilidad familiar [en línea]. Disponible en: <http://dialnet.unirioja.es/ref/4bl6> [Consulta: 22 de septiembre de 2014].

23 Bibliografía sobre Prevención de la violencia de género [en línea]. Disponible en: <http://dialnet.unirioja.es/ref/x96N> [Consulta: 22 de septiembre de 2014]

${ }^{24}$ El Ranking web de Repositorios [en línea]. Disponible en: <http://repositories.webometrics.info/es/top_portales> [Consulta: 22 de septiembre de 2014].

25 Dialnet se consolida como el segundo portal de contenidos bibliográficos del mundo (11/02/2013) [en línea]. Disponible en: $<$ http://dialnet.unirioja.es/servlet/noticia?codigo=217> [Consulta: 22 de septiembre de 2014].

\section{BIBLIOGRAFÍA}

AGUILERA, R. et al. Enseñando a buscar y a utilizar información estadística: una colaboración conjunta entre docentes y personal de biblioteca [en línea]. En: World library and information congress: 76th IFLA general conference and assembly, 2010, p. 10-15. Disponible en: <http://conference.ifla.org/past-wlic/2010/86-aguileraes.pdf $>$ [Consulta: 22 de septiembre de 2014].

AREA, M. De la biblioteca universitaria al centro de recursos para el aprendizaje e investigación, informe final [en línea]. Madrid: Dirección General de Universidades, 2004. Disponible en: <http://www.ub.edu/esbrina/docs/areacrai.pdf> [Consulta: 22 de septiembre de 2014].

ARÉVALO-BAEZA, M.; DÓMINGUEZ-AROCA, M.I. e IGLESIA SÁNCHEZ, A. de la. Trabajo colaborativo e interdisciplinar para el desarrollo de competencias transversales informacionales: una experiencia en el Grado de Ciencias de la Actividad Física y del Deporte [en línea]. En: V Encuentro de Innovación en Docencia Universitaria, Alcalá de Henares, 2011. Disponible en: <http://eprints.rclis.org/15565/> [Consulta: 22 de septiembre de 2014].

CHAHER, S. y SANTORO, S. (Comp.). Las palabras tienen sexo. Introducción a un periodismo con perspectiva de género [en línea]. Buenos Aires, Artemisa Comunicación Ediciones, 2007. Disponible en: <http://www.artemisanoticias.com.ar/images/las-palabras-tienen-sexo.pdf> [Consulta: 22 de septiembre de 2014]. 
CIVILA SALAS, A. La responsabilidad y la corresponsabilidad como valor educativo [en línea]. En: XII Congreso Internacional de Teoría de la Educación, Barcelona, 2011. Disponible en: $<$ http://www.cite2011.com/Comunicaciones/A+R/055.pdf> [Consulta: 22 de septiembre de 2014].

Asociación Transnacional EQ-MIND, recopilación. Guía práctica para la planificación de acciones formativas en materia de género e igualdad de oportunidades [en línea]. 2004. Disponible en: <http://www.empleo.gob.es/uafse_2000-2006/equal/ProductosEqual/archivos/AD_498_Producto_1.pdf> [Consulta: 22 de septiembre de 2014].

Los Centros de Recursos para el Aprendizaje y la Investigación en los procesos de innovación docente, Jornadas REBIUN, Palma de Mallorca [en línea]. 2003. Disponible en: <http://biblioteca.uam.es/sc/palma.html> [Consulta: 22 de septiembre de 2014].

COMISIÓN EUROPEA. El papel de las universidades en la Europa del conocimiento. Comunicación de la Comisión COM/2003/58 final [en línea]. 2003. Disponible en: <http://eurlex.europa.eu/LexUriServ/LexUriServ.do?uri=COM:2003:0058:FIN:ES:PDF> [Consulta: 22 de septiembre de 2014].

ELÓSEGUI ITXAXO, M. Educar en la corresponsabilidad entre mujeres y hombres: la igualdad necesaria [en línea]. Revista General de Derecho Canónico y Derecho Eclesiástico del Estado, 2009, nº 20.

ESPÍN LÓPEZ, J.V. Educación, ciudadanía y género. En: M. Bartolomé (Coord.) Identidad y ciudadanía. Un reto a la educación intercultural. Madrid: Narcea, 2002, p. 105-130.

GARCÍA NIETO, M.T. y LEMA DEVESA, C. Guía de intervención ante la publicidad sexista [en línea]. Madrid: Ministerio de Igualdad. Instituto de la Mujer, 2008. Disponible en: <http://www.inmujer.gob.es/observatorios/observIgualdad/estudiosInformes/docs/012-guia.pdf> [Consulta: 22 de septiembre de 2014].

GONZÁLEZ, J. y WAGENAAR, R., (eds.) Tuning educational structures in Europe. Bilbao: Universidad de Deusto, 2003.

KAGAN, A. IFLA and Social Responsibility: A Core Value of Librarianship [en línea]. En: Seidelin, S. and Hamilton, S. (eds.) Libraries, National Security, Freedom of Information Laws and Social Responsibilities: IFLA/FAIFE World Report. Copenhagen: IFLA/FAIFE, 2005, p. 33-43. Disponible en: $<$ http://www.indiana.edu/ libsalc/african/IFLA.pdf> [Consulta: 22 de septiembre de 2014].

LEÓN, J.L. Los efectos de la publicidad. Barcelona: Ariel Comunicación, 1996.

LÓPEZ YEPES, J. El nuevo profesional de la información, del conocimiento y de la comunicación: el bibliotecario universitario [en línea]. Anales de documentación, 2007, $\mathrm{n}^{\circ}$ 10, p. 263-279. Disponible en: $<$ http://digitum.um.es/xmlui/bitstream/10201/4022/3/1241.pdf> [Consulta: 22 de septiembre de 2014].

MAILLARD MANCILLA, C. et al. Guía para la incorporación del enfoque de género en bibliotecas [en línea]. Santiago de Chile: DIBAM, Dirección de Bibliotecas, Archivos y Museos, 2012. Disponible en: <http://www.germina.cl/wp-content/uploads/2012/10/guia_incorporacion_enfoque_genero_bibliotecas.pdf> [Consulta: 22 de septiembre de 2014].

MARRAUD, G. Las bibliotecas y la responsabilidad social universitaria: informe de REBIUN [en línea]. En: XIII Jornadas Españolas de Documentación FESABID 2013, Toledo, 24 y 25 mayo 2013. Disponible en: $<$ http://eprints.rclis.org/20107/> [Consulta: 22 de septiembre de 2014].

MARTÍNEZ, D. El Centro de Recursos para el Aprendizaje CRAI: El nuevo modelo de biblioteca universitaria [en línea]. Material presentado al curso de formación "Las tecnologías de la información como recurso estratégico y para el establecimiento de políticas directivas", organizado por la Universidad Complutense de Madrid, septiembre de 2004. Disponible en: <http://upcommons.upc.edu/e-prints/handle/2117/11982> [Consulta: 22 de septiembre de 2014].

MOSCOSO, P. La nueva misión de las bibliotecas universitarias ante el Espacio Europeo de Enseñanza Superior [en línea]. En: Los Centros de Recursos para el Aprendizaje y la Investigación en los procesos de innovación docente, Jornadas REBIUN, Palma de Mallorca, 2003. Disponible en: <http://hdl.handle.net/10017/809> [Consulta: 22 de septiembre de 2014].

NAVARRO BELTRÁ, M. y MARTÍN LLAGUNO, M. La publicidad sexista en España. Eficacia de la Ley Orgánica de Medidas de Protección Integral contra la Violencia de Género. Cuestiones de género: de la igualdad y la diferencia, 2012, $\mathrm{n}^{\circ}$ 7, p. 247-267.

PEÑA PALACIOS, E.M. de la. Violencia de género [en línea]. Mancomunidad de Municipios Valle del Guadiato. 2007. Disponible en: <http://www.fundacionmujeres.es/maletincoeducacion/pdf/CUAD5horiz.pdf> [Consulta: 22 de septiembre de 2014].

ROYO, M. et al. Roles de género y sexismo en la publicidad de las últimas revistas españolas. Un análisis de las tres últimas décadas del siglo XX [en línea]. Comunicación y Sociedad, 2005, vol. 18, nº 1, p. 113-152. Disponible en: <http://www.unav.es/fcom/comunicacionysociedad/es/articulo.php?art_id=69> [Consulta: 22 de septiembre de 2014]. 
SANTANA HIDALGO, A. et al. Experiencia de colaboración entre docentes y personal de biblioteca en la EPS de Sevilla para la integración de competencias informacionales [en línea]. En: Retos y oportunidades del desarrollo de los nuevos títulos en educación superior, VIII Jornadas de Innovación Universitaria, Villaviciosa de Odón, 2011. Disponible en: <http://hdl.handle.net/11268/1551> [Consulta: 22 de septiembre de 2014].

TORREGROSA CARMONA, J.F. Comunicación periodística. Notas desde una perspectiva de género. Feminismo/s: Revista del Centro de Estudios sobre la Mujer de la Universidad de Alicante, 2008, nº 11, p. 125-140. 